\title{
Aplicabilidad de paradigmas duales cognitivo-motrices a una muestra de personas mayores institucionalizadas
}

\author{
D. Facal ${ }^{\mathrm{a}}$, L. Lorenzo-López ${ }^{\mathrm{b}}$, R. López-López ${ }^{\mathrm{b}}$, J.C. Millán-Calenti ${ }^{\mathrm{b}}$, A.X. Pereiro ${ }^{\mathrm{a}}$,
} J.M. Cancela ${ }^{\mathrm{c}}$

${ }_{\text {a Universidade de Santiago de Compostela, Departamento de Psicoloxía Evolutiva e da Educación, Santiago de }}$ Compostela, La Coruña, España
${ }^{b}$ Universidade da Coruña, Grupo de Investigación en Gerontología, Instituto de Investigación Biomédica de A
Coruña (INIBIC), Complexo Hospitalario Universitario de A Coruña (CHUAC), SERGAS, A Coruña, España
${ }^{c}$ Universidade de Vigo, Grupo de Investigación HealthyFit. Instituto de Investigación Sanitaria Galicia Sur (IISGS),
Complejo Hospitalario Universitario de Pontevedra (CHOP), SERGAS, Vigo, Pontevedra, España

\begin{abstract}
Objetivos: Las tareas duales miden la capacidad de los individuos para combinar la ejecución concurrente de varias tareas y repartir los recursos cognitivos para realizarlas. Cuando las exigencias de las tareas exceden los recursos disponibles, se produce un coste de respuesta con respecto del rendimiento en la tarea en solitario. El rendimiento en tareas duales se ha relacionado con la velocidad de la marcha y el riesgo de caídas, y en los últimos años con la fragilidad cognitiva. El objetivo del presente trabajo es probar un paradigma de tareas duales que permita analizar el coste de respuesta tanto cognitivo como motriz.
\end{abstract}

Método: Se realizó un estudio transversal con una muestra de 33 personas de 65 o más años, institucionalizadas en un complejo gerontológico con un GDS de 2, 3 o 4. Se realizó una valoración geriátrica integral que incluía, entre otras la evaluación del fenotipo de fragilidad mediante criterios de Fried y el estado cognitivo mediante el Mini Mental State Examination. Complementariamente, se aplicaron tres tareas simples (fluidez fonológica, velocidad visuomotriz y pedaleo, esta última con tren superior e inferior) de una duración de $1 \mathrm{~min}$, y tres tareas duales combinando la fluidez fonológica con las tareas motrices. A través de chi cuadrado se comparó la aplicabilidad de las tareas duales (aplicable / no aplicable) en los grupos según el estatus cognitivo (GDS) y según la situación física (pre-frágil y frágil).

Resultados: Se obtuvieron altos porcentajes de respuesta para las tareas de fluidez (93,9\%), velocidad visuomotriz $(87,9 \%)$, pedaleo con tren superior $(93,9 \%)$ y pedaleo con tren inferior $(78,8 \%)$. Respecto a las tareas duales, se obtuvieron buenos porcentajes de respuesta de la tarea dual-visuoespacial $(78,8 \%)$ y dual-pedaleo de tren superior $(81,8 \%)$, y menores de dual-tren inferior $(57,6 \%)$. No se encontraron diferencias en el porcentaje de aplicación entre los grupos según el estatus cognitivo ni el grado de fragilidad.

Conclusiones: Los paradigmas duales que combinan fluidez fonológica con velocidad visuomotriz y pedaleo del tren superior tienen buena aplicabilidad en una muestra de personas mayores institucionalizada.

Este trabajo ha sido financiado por la Xunta de Galicia (ED431C-2017/27, ED431C 2017/49 y Red FrailNet IN607C 2016/08). 